\title{
An efficient fringe integral equation method for optimizing the antenna location on complex bodies
}

Jørgensen, Erik; Meincke, Peter; Breinbjerg, Olav

Published in:

Proceedings of IEEE Antennas and Propagation Society International Symposium

Link to article, DOI:

10.1109/APS.2001.959791

Publication date:

2001

Document Version

Publisher's PDF, also known as Version of record

Link back to DTU Orbit

Citation (APA):

Jørgensen, E., Meincke, P., \& Breinbjerg, O. (2001). An efficient fringe integral equation method for optimizing the antenna location on complex bodies. In Proceedings of IEEE Antennas and Propagation Society

International Symposium (Vol. 2, pp. 584-587). IEEE. https://doi.org/10.1109/APS.2001.959791

\section{General rights}

Copyright and moral rights for the publications made accessible in the public portal are retained by the authors and/or other copyright owners and it is a condition of accessing publications that users recognise and abide by the legal requirements associated with these rights.

- Users may download and print one copy of any publication from the public portal for the purpose of private study or research.

- You may not further distribute the material or use it for any profit-making activity or commercial gain

- You may freely distribute the URL identifying the publication in the public portal 


\section{An Efficient Fringe Integral Equation Method for} Optimizing the Antenna Location on Complex Bodies

\section{Erik Jørgensen, Peter Meincke, and Olav Breinbjerg}

Technical University of Denmark

Ørsted.DTU, Section for Electromagnetic Systems

$\emptyset$ rsteds Plads, Bldg. 348, DK-2800 Kgs. Lyngby, Denmark ej@oersted.dtu.dk

\section{Introduction}

The radiation pattern of an antenna mounted nearby, or directly on, a complex three-dimensional (3D) structure can be significantly influenced by this structure. Integral equations combined with the method of moments (MoM) provide an accurate means for calculating the scattering from the structures in such applications. The structure is then modelled by triangular or rectangular surface patches with corresponding surface current expansion functions. A monopole antenna mounted on the structure may be modelled by a wire segment with corresponding line current expansion functions. This modelling has the drawback, that the MoM matrix depends in part on the antenna location. Hence, this part of the MoM matrix must be recalculated and the equation system re-solved if the antenna location is changed. In some applications, e.g., if the optimum location of a flush-mounted antenna on a satellite must be determined, this recalculation and re-solving for every antenna location poses a major computational problem.

A MoM matrix which is independent of the antenna location can be obtained by modelling the antenna as an impressed electric or magnetic source, e.g., a slot antenna can be modelled by a magnetic Hertzian dipole. For flush-mounted antennas, or antennas mounted in close vicinity of the scattering structure, the nearby impressed source induces a highly peaked surface current on the scattering structure. For the low-order basis functions usually applied in conventional integral equation solvers, a peaked current poses a challenging problem since it necessitates a large number of unknowns and excessive computation times. A fringe dual-surface magnetic field integral equation (F-DMFIE) that eliminates the problem of peaked currents and fields, even for impressed sources located arbitrarily close to the surface of the structure, was presented in [1]. In this formulation, the surface current on the structure is obtained by evaluating a number of line integrals and performing a single matrix-vector multiplication for each antenna location. This paper briefly reviews the F-DMFIE formulation and applies it to a more complicated geometry than in [1]. In addition, efficient solution methods for multiple antenna locations, including parallel implementations, are discussed.

2. The Fringe Dual-Surface Magnetic Field Integral Equation The magnetic field integral equation (MFIE) for a closed PEC body is often expressed as

$$
\mathbf{J}(\mathbf{r})+2 \hat{\mathbf{n}} \times f_{S} \mathbf{J}\left(\mathbf{r}^{\prime}\right) \times \nabla G\left(\mathbf{r}, \mathbf{r}^{\prime}\right) d S^{\prime}=2 \hat{\mathbf{n}} \times \mathbf{H}^{i n c}(\mathbf{r})
$$

where the bar on the integral sign denotes a principal value integral, $\mathbf{r}$ and $\mathbf{r}^{\prime}$ are the observation and integration points, respectively, $\mathbf{J}$ is the unknown surface 
current density, $\hat{\mathbf{n}}$ is an outward normal vector at the point of observation, and $G\left(\mathbf{r} . \mathbf{r}^{\prime}\right)$ is the free-space 3D scalar Green function, $G\left(\mathbf{r}, \mathbf{r}^{\prime}\right)=\frac{\exp \left(-j k\left|\mathbf{r}-\mathbf{r}^{\prime}\right|\right)}{4 \pi\left|\mathbf{r}-\mathbf{r}^{\prime}\right|}$, with $k$ being the wavenumber and an assumed time-dependence $\exp (j \omega t)$. Due to the current peak, as well as other problems discussed in [1], a conventional solution to (1) is troublesome if the incident field is caused by an impressed source in close vicinity of $S$, e.g., a Hertzian dipole. To overcome these difficulties, we subtract the known PO current from the total current in some region close to the source [2]. We denote this PO region by $S^{p o}$ which is the largest planar surface close to the source. We define $\mathbf{J}^{p o}=2 \hat{\mathbf{n}} \times \mathbf{H}^{i n c}$ inside $S^{p o}$ and $\mathrm{J}^{p o}=0$ outside $S^{p o}$. The total current is separated in this PO part and the remaining fringe part, i.e., $\mathbf{J}=\mathbf{J}^{p o}+\mathbf{J}^{f}$. Note that the region directly illuminated by the source might be larger than the PO region used here; hence, the present definition of $J^{p o}$ may differ from the usual one. By introducing this separation in the MFIE (1) we obtain the fringe magnetic field integral equation (F-MFIE)

$$
\begin{aligned}
& \mathbf{J}^{f}(\mathbf{r})+2 \hat{\mathbf{n}} \times f_{S} \mathbf{J}^{f}\left(\mathbf{r}^{\prime}\right) \times \nabla G\left(\mathbf{r}, \mathbf{r}^{\prime}\right) d S^{\prime}= \\
& 2 \hat{\mathbf{n}} \times \mathbf{H}^{i n c}(\mathbf{r})-\mathbf{J}^{p o}(\mathbf{r})-2 \hat{\mathbf{n}} \times f_{S^{p o}} \mathbf{J}^{p o}\left(\mathbf{r}^{\prime}\right) \times \nabla G\left(\mathbf{r}, \mathbf{r}^{\prime}\right) d S^{\prime} .
\end{aligned}
$$

This equation is more easily solved than (1) for a source close to the scatterer On the left-hand side we encounter the original MFIE but now with the fringe current as the unknown. Thus, the MoM-matrix used in the solution of (2) is the same as in (1). The entire right-hand side is zero inside the PO region but the first and the last term contribute outside. The first term is easily evaluated since the incident magnetic field is well-behaved outside the PO region. The last term is the magnetic field radiated by the PO current. Evaluating this directly by a surface integration will be very time-consuming due to the highly peaked integrand. However, the field radiated by the PO current on a planar PEC plate illuminated by a Hertzian dipole can be rigorously transformed into a line integral along the edges of the plate [3]. This involves only a one-dimensional integral and the incident field at the edges of the PO region, where it is well-behaved. For the reasons explained in [1], a dual-surface formulation [4] has been preferred to eliminate the interior resonance problem of a closed PEC body. This formulation is easily implemented and involves only the electric current and the magnetic field as in the ordinary MFIE.

3. Solution Method for Multiple Antenna Locations

By using an impressed source and the F-DMFIE formulation, the solution involves a MoM matrix independent of the antenna location and well-behaved currents and fields. The matrix equation could be solved with an iterative solver. However, if many antenna locations must be investigated a direct solver will be faster since the inverse of the MoM matrix is obtained. For each antenna location, the right-hand side of (2) is obtained by evaluating a line integral for all observation points outside the PO region. The unknown surface current is then found by multiplying the right-hand side with the inverted MoM matrix. This scheme will be efficient when a large number of antenna locations is to be investigated.

A full matrix inversion requires large computer resources, which calls for a parallel implementation, preferably on a low-cost distributed memory system. The 
filling of the MoM matrix and the calculation of the right-hand sides can easily be parallelized, and efficient implementations exist for matrix inversion on distributed memory systems [5]. The F-DMFIE has been implemented in a scattering code for general 3D bodies using the well-known triangular-cell RWG basis functions [6]. This code runs on a Linux cluster with 16 PC's using the message passing interface (MPI) standard for communication

\section{Numerical Example}

We consider now the PEC structure shown in Figure 1 with an associated rectangular coordinate system as indicated in the figure. The structure can be viewed as a $3 \lambda \times 2 \lambda \times 1 \lambda$ box with another $1 \lambda \times 2 \lambda \times 1 \lambda$ box on top. The structure is excited by a $y$-directed magnetic Hertzian dipole located very close to the surface at $\left(0,0, \frac{\lambda}{50}\right)$ which will result in a highly peaked current. With a sufficiently high basis function density, say $700 / \lambda^{2}$, a conventional dual-surface MFIE and the F-DMFIE converge to the same result. The total surface current obtained with DMFIE is shown in Figure 1(a) where the current has been normalized to the maximum value occurring at the peak and the scale is in $\mathrm{dB}$. It is observed, that the current on the major part of the structure is more than $60 \mathrm{~dB}$ lower than the value at the peak. Thus, the configuration is well suited for the F-DMFIE. The fringe current obtained with this formulation is shown in Figure 1(b). The $\mathrm{PO}$ region is in this case chosen to be the horizontal $2 \lambda \times 2 \lambda$ planar plate with center at the origin of the coordinate system. Hence, the vertical plate defined by $(x=0,-1 \lambda<y<1 \lambda, 0<z<1 \lambda)$ is not a part of the PO region although it is directly illuminated by the source. Note that the current peak at the source in Figure 1(a) is absent in Figure 1(b) since the peak is entirely contained in the PO current. Also note that the fringe current is discontinuous across the line $(x=1 \lambda,-1 \lambda<y<1 \lambda, z=0)$. It was demonstrated for a similar configuration in [1], that the F-DMFIE is converged with a basis function density of approximately $100 / \lambda^{2}$ whereas the DMFIE needs more than $700 / \lambda^{2}$. The high basis function density in the DMFIE is required to model the highly peaked total surface current while the F-DMFIE is capable of modelling the well-behaved fringe current with far fewer basis functions.

5. Conclusions

A F-DMFIE formulation was reviewed. The formulation is useful when impressed electric or magnetic sources are located arbitrarily close to a PEC body This creates a highly peaked surface curtent that hampers an efficient solution by the standard EFIE/MFIE formulations. The F-DMFIE formulation uses only well-behaved currents and fields and is therefore well-suited for a numerical solution. By using this formulation, an antenna can be modelled by an impressed source which makes the MoM matrix independent of the antenna location. Thus, the F-DMFIE is an efficient method for optimizing the antenna location on a complex body.

\section{References}

[1] E. Jørgensen, P. Meincke, and O. Breinbjerg, "A fringe dual-surface magnetic field integral equation for three-dimensional structures with nearby sources." in Proc: of the Applied Computational Electromagnetics Symp., Monterey, CA. March 2001.

[2] E. Jørgensen, S. Maci, and A. Toccafondi, "Fringe integral equation method for a truncated grounded dielectric slab," to appear in IEEE Trans. Antennas Propagat. 


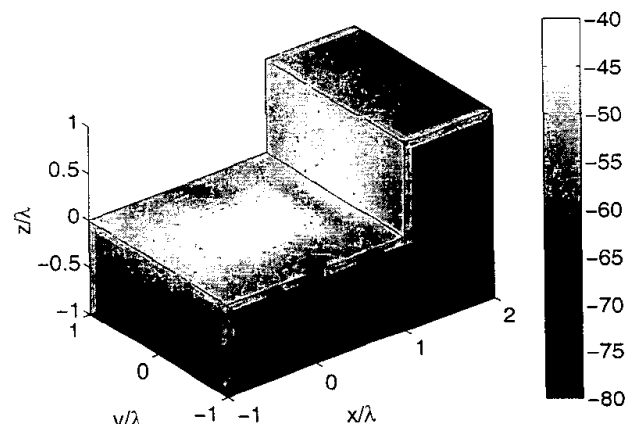

(a)

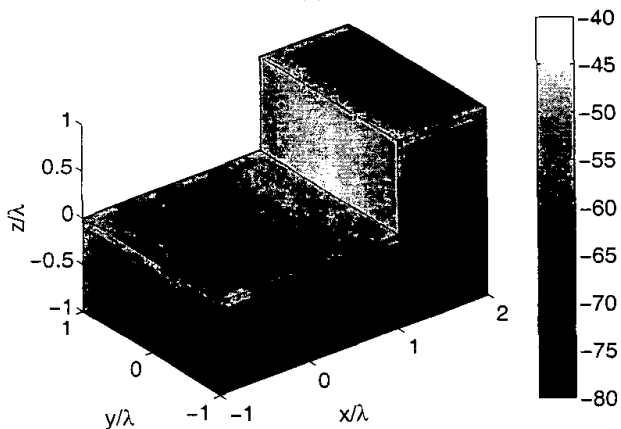

(b)

Figure 1: (a) Total current obtained with DMFIE and (b) fringe current obtained with $F$-DMFIE. The scale is in $d B$ normalized with respect to the current maximum occurring at the peak.

vol. 49, no. 3, March 2001.

[3] P. Meincke, O. Breinbjerg, and E. Jørgensen, "The magnetic physical optics scattered field in terms of a line integral," in Proceedings of the 2000 IEEE Antennas and Propagation Soc. Int. Symp'., Salt Lake City, UT, July 2000, vol. 1, pp. 402-405.

[4] M. B. Woodworth and A. D. Yaghjian, "Multiwavelength three-dimensional scattering with dual-surface integral equations," Jour. Opt. Soc. Am. A, vol. 11, no. 4 pp. 1399-1413, April 1994.

[5] ScaLAPACK Users' Guide, SIAM, Philadelphia, 1997.

[6] S. M. Rao, D. R. Wilton, and A. W. Glisson, "Electromagnetic scattering by surfaces of arbitrary shape," IEEE Trans. Antennas Propagat., vol. AP-30, no. 3, pp. 409 418, May 1982. 